\title{
CONTROL DE TEMPERATURA PARA UNA MUFLA UTILIZANDO EL PLC MOLLER EC4P-200
}

(Temperature control for a muffle oven using the PLC Moller Ec4p-200)

Iván Manuel Fernández Betancourt

Universidad Pedagógica y Tecnológica de Colombia. Grupo de Investigación DSP

Correo: ivanfernandez10@msn.com.

(Recibido 19 Junio de 2012 y Aceptado 13 de Marzo de 2013)

\begin{abstract}
Resumen:
El artículo presenta el diseño y la implementación de un sistema de control de temperatura en un horno tipo mufla, perteneciente al laboratorio de carbones de la UPTC seccional Sogamoso. Se usa el módulo didáctico PLC EC4P del laboratorio de automatización industrial de la Escuela de Ingeniería Electrónica de la misma institución. Se describe el procedimiento realizado utilizando los elementos que conforman el módulo, como el PLC Moller EC4P-200, pantalla táctil HMI y transmisores universales de temperatura para acondicionar la señal de la termocupla tipo K, sensor utilizado para la medición de la temperatura en el interior de la mufla. Además se muestra el modelado del sistema y el diseño del controlador PID empleado, así como los resultados obtenidos de la implementación del controlador en el horno.
\end{abstract}

Palabras clave: mufla, PID, PLC, termocupla, transmisor de temperatura.

\section{Abstract:}

This report presents the design and implementation of a temperature control system in a muffle oven belonging to the coal laboratory of UPTC Sogamoso. We use the PLC EC4P didactic module of the Industrial Automation Laboratory of the School of Electronic Engineering from the same institution. This paper describes the procedure performed using the elements of the module, such as the PLC Moller EC4P-200, HMI touch screen and temperature universal transmitters to adjust the signal from the type $\mathrm{K}$ thermocouple which is the sensor used to measure the temperature inside of the muffle. Besides, it is shown the system modeling and the design of a PID controller as well as the obtained results of the controller implementation on the oven.

Keywords: muffle, PID, PLC, thermocouple, temperature transmitter.

\section{INTRODUCCIÓN}

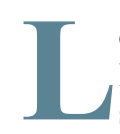
os procesos térmicos siempre han estado presentes a lo largo de nuestra historia, han contribuido de manera significativa en los avances tecnológicos y se han utilizado en innumerables aplicaciones tanto domésticas como industriales. Entre los procesos industriales más comunes relacionados con el uso de la energía térmica, se destacan la generación de energía, fabricación de alimentos, elaboración de productos farmacéuticos y manufactura de componentes.

En el ámbito académico, en la Universidad Pedagógica y Tecnológica de Colombia, los procesos térmicos son utilizados en el estudio y análisis de materiales, principalmente en el laboratorio de carbones donde se analiza el mineral proveniente de las diferentes minas de la región. En las labores diarias del laboratorio es muy común el uso de los hornos tipo mufla para hacer pruebas de volatilidad y de cenizas al carbón aplicando diferentes temperaturas.

Es de vital importancia suministrar la temperatura adecuada para cada tipo de análisis y evitar el malogramiento de las muestras que se refleja en la alteración adversa de los resultados de los estudios.
Las muflas generalmente cuentan con un sistema de control analógico mediante temporizado para regular la temperatura deseada. Actualmente se han venido implementando en ellas controles digitales de temperatura que permiten mayor exactitud, facilitando su uso para el usuario, pues proveen información adicional como visualizaciones y versatilidad en su configuración. Pero debido a su uso continuo, estos dispositivos presentan fallas y averías, haciendo que las muflas estén fuera de operación por un largo tiempo hasta ser reparadas.

En este trabajo se propone una alternativa al control de los hornos que están fuera de operación debido a su antigüedad y uso continuo, utilizando el equipo existente en el laboratorio de electrónica, esto es, los módulos de automatización EC4P, además del diseño e implementación de la instrumentación necesaria para rehabilitar estos equipos. El controlador elegido para el desarrollo de esta aplicación fue de tipo PID, ya que este se encuentra disponible en las funciones internas del PLC Moller, componente fundamental del módulo mencionado anteriormente. Asimismo, se llevó a cabo el modelado y la identificación de todo el sistema de temperatura para una zona de operación específica, y se hizo el diseño a partir de esta información. 
Antes de realizar el diseño, se buscó información concerniente al tema del control de la temperatura aplicado a sistemas de hornos y elementos relacionados con sistemas calefactores, para esbozar de una manera general la metodología que se sigue en el diseño y la implementación del sistema de control. Entre las más relevantes se destaca la aplicación desarrollada en la Escuela Politécnica Nacional del Ecuador, donde se hizo la programación e instalación de un sistema de control de supervisión y adquisición de datos de un horno tipo mufla Lindberg del laboratorio de tratamientos térmicos, utilizando un PLC (Herrera, Velázquez \& Estalin, 2011). De igual manera, en la Universidad Nacional Autónoma de México UNAM, se diseñó e implementó un controlador PID de temperatura de tipo didáctico, regulando la temperatura del agua utilizando un sistema simple y de bajo costo basado en una parrilla eléctrica y un circuito integrado como elemento sensor de la temperatura (Tarazona, 2008). Por otra parte, en la Universidad Nacional Experimental del Táchira en Venezuela, el Departamento de Ingeniería Electrónica diseñó y construyó un controlador de temperatura tipo rampa, para el laboratorio de catálisis en el proceso de análisis cromatográfico, utilizando un horno calefactor y un controlador PID como estrategia de control. El sistema abarcó el control de la rampa de la temperatura, así como la regulación del tiempo transcurrido mediante la variación de la corriente eficaz entregada a la resistencia del horno (Bañuelos et al., s.f.).

\section{DESCRIPCIÓN DELSISTEMA}

El sistema propuesto para el control de temperatura del horno tipo mufla, consta de los bloques que se observan en la Figura 1.

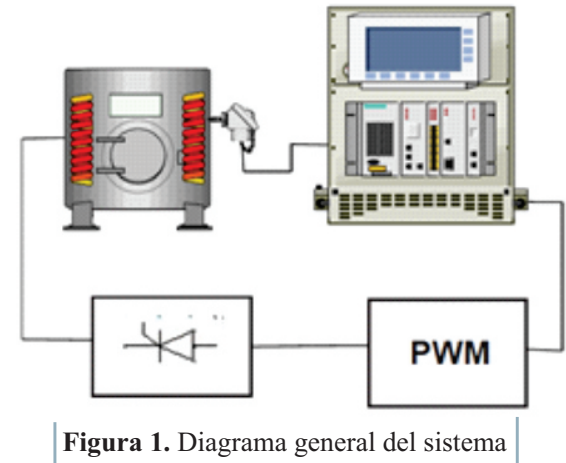

El primer componente del sistema es el horno tipo mufla, el cual cuenta con una resistencia eléctrica como elemento calefactor y una termocupla tipo $\mathrm{k}$ en su interior, para la medición de la temperatura. El segundo componente es el modulo didáctico EC4P, en el cual se realiza el acondicionamiento de las señales y se encuentra el PLC, que es el encargado de controlar el sistema. Además se encuentra la pantalla HMI con la cual se accede a la supervisión del proceso, parámetros de configuración del controlador, y visualización, entre otras opciones. El siguiente componente es el actuador del sistema, que permite aplicar las señales de control enviadas desde el PLC, compuesto por un corte de fase monofásico con dos SCRs en antiparalelo, el cual varía el voltaje aplicado a la resistencia calefactora de la mufla mediante una modulación por ancho de pulso PWM, aplicada a la compuerta de los semiconductores.

\subsection{Horno de mufla}

El objeto del sistema de control es un horno tipo mufla (ver Figura 2). Básicamente, la mufla en su estructura cuenta con una cámara de cocción donde se almacenan las muestras bajo estudio. Las paredes internas están construidas con material refractario y material aislante que permiten contener el calor dentro de la misma. El sistema de calefacción del horno es una resistencia eléctrica incrustada en las paredes laterales de la cámara interna, que logra alcanzar temperaturas entre los 1200 a 1500 grados centígrados. La puerta de acceso a la cámara central cuenta con un agujero que permite observar el interior de esta y contribuye a la expulsión de gases que se puedan formar en el tratamiento de las diferentes muestras (Luengo, 2003).

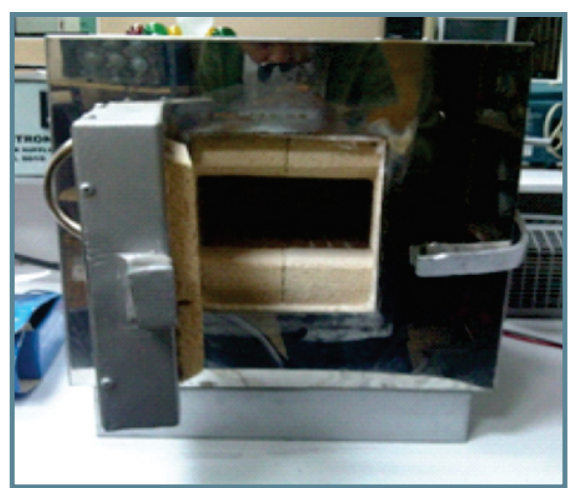

Figura 2. Horno tipo mufla empleado para diseñar e implementar el sistema de control de temperatura.

\subsection{Módulo didáctico Ec4p}

El módulo didáctico EC4P, disponible en el laboratorio de la Escuela de Ingeniería Electrónica de la UPTC, y que se utilizó para controlar la temperatura (ver Figura 3), cuenta en su parte inferior con borneras para entradas y salidas tanto digitales como análogas, dos transmisores universales programables de temperatura vía USB con salida 4 a $20 \mathrm{~mA}$. En su panel frontal se encuentran diversas luces pilotos, indicadores, selectores y una pantalla táctil HMI XV200, con programación vía Ethernet y comunicación CAN open. En su parte interna hay una fuente de alimentación de $24 \mathrm{~V}$, un PLC Moller que tiene doce entradas digitales, de las cuales cuatro pueden ser configuradas como entradas análogas, ocho salidas a transistor y una salida analógica. Además tiene una serie de relevos para la conexión a cargas de mayor voltaje y amperaje (BP, 2011).

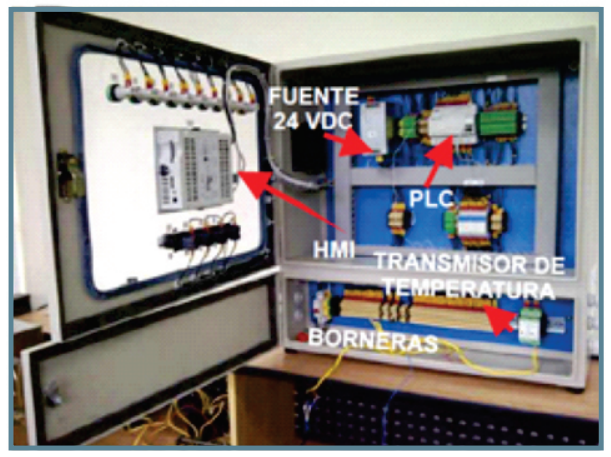

Figura 3. Módulo didáctico EC4P, vista interna.
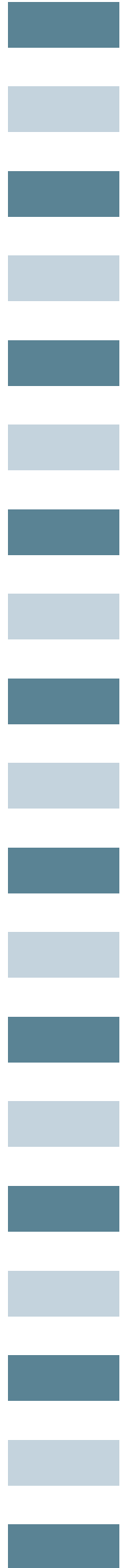
En la parte lateral izquierda del módulo hay conectores RJ45, para la comunicación del protocolo CAN open, así como conectores para la programación Ethernet del PLC y la pantalla HMI.

\subsection{Sistema de medición}

El sensor utilizado para la medición de la temperatura dentro de la cámara de la mufla, es una termocupla tipo $\mathrm{K}$ capaz de efectuar mediciones hasta los 1200 grados centígrados aproximadamente (Martínez \& Azuaga, 1997). Esta presenta su juntura expuesta haciéndola mucho más sensible a los cambios de temperatura, pero, a la vez, acortando su vida útil al estar en contacto directo con las altas temperaturas.

\subsubsection{Patronamiento y acondicionamiento}

Fue necesario hacer un patronamiento del sensor de temperatura, para garantizar una adecuada medición de la misma. Para lograrlo, se utilizó un termómetro de mercurio como elemento patrón, con el que se hicieron mediciones de temperatura y voltaje generados en la unión de la termocupla al estar en contacto con una superficie caliente. Las muestras se tomaron con intervalos de 5 grados centígrados, de forma tanto ascendente como descendente, obteniendo como resultado que por cada aumento o decremento de 5 grados centígrados en temperatura, el incremento o decremento del voltaje en la unión fue aproximadamente de $2 \mathrm{mV}$.

Como el voltaje generado por la termocupla es muy pequeño, generalmente del orden de los $\mathrm{mV}$, es necesario acondicionarlo a niveles más apropiados para su uso. Para ello se utilizó un transmisor de temperatura universal Intech XU2, disponible en el módulo didáctico de PLC EC4P(Intech, 2011). Pero debido a que el PLC en su configuración no posee entradas de 4 a $20 \mathrm{~mA}$, el módulo internamente convierte la señal de corriente a una señal de voltaje en el rango de 0 a $10 \mathrm{~V}$, y así puede ser adquirido por medio de las entradas analógicas.

\subsection{Actuador del sistema}

El actuador utilizado en el diseño y la implementación fue un corte de fase monofásico con dos SCRs Semikron SKKT15, encapsulados en un mismo módulo con capacidad máxima de 24 A (Semikron, 2004). El SCR proporciona una técnica apropiada para el control del voltaje promedio que se aplica a una carga, cambiando el ángulo de fase al que se suministra el voltaje de alimentación de dicha carga. Para controlar el ángulo de fase, es necesario regular el ancho de pulso del disparo que activa el SCR (Chapman, 1993). Los dos SCRs se utilizaron en una configuración antiparalela (ver Figura 4), para aprovechar al máximo cada semiciclo de la red eléctrica y garantizar así mayor voltaje en la carga.

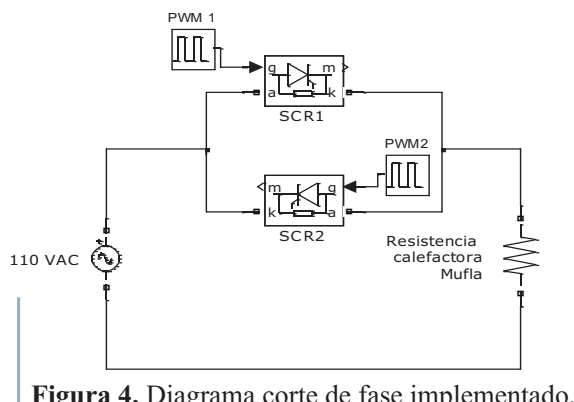

Para generar los disparos de los SCRs, se utilizó un PWM (Pulse-width modulation) construido a partir de amplificadores operacionales, y sincronizado con la red eléctrica $(60 \mathrm{~Hz})$. Se generaron dos PWM diferentes, uno desfasado 180 grados respecto al otro, para realizar el disparo de los SCRs, uno por cada semiciclo de la red eléctrica.

Cabe destacar que el circuito generador del PWM se encuentra aislado eléctricamente del circuito de potencia (corte de fase), mediante opto-acopladores MOC 3021, y el acho de pulso se acondicionó de 0 y $10 \mathrm{~V}$, donde $0 \mathrm{~V}$ es el $100 \%$ del ciclo útil, y $10 \mathrm{~V}$ equivale al $0 \%$ del ancho del pulso. Después de realizar el montaje del corte de fase, junto con los PWM se obtuvo como resultado en la carga, la señal de la Figura 5, donde se muestra un ángulo de disparo cercano a los $180^{\circ}$.

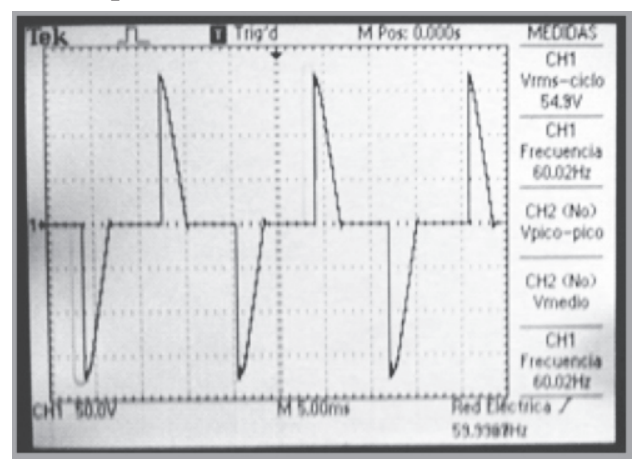

Figura 5. Señal obtenida en la carga a la salida del corte de fase monofásico con un ángulo cercano a los $180^{\circ}$.

\section{MODELADO}

Habiendo logrado la puesta en marcha del sistema de temperatura, se procedió a encontrar experimentalmente un modelo que represente el comportamiento del sistema en una región de operación. La elección para ello fue el método por curva de reacción, utilizando una tarjeta de adquisición de datos National Instruments NI 6211.

El punto de operación seleccionado fue los $320^{\circ} \mathrm{C}$, que es la temperatura que alcanza la mufla al excitar las resistencia con el $50 \%$ del ancho de pulso del PWM. Una vez estabilizada la temperatura en este punto, se aplica un aumento en el ancho del pulso del PWM, alcanzando una temperatura de aproximadamente $700^{\circ} \mathrm{C}$. Estos cambios se registran mediante la tarjeta de adquisición de datos. Se registró tanto la señal de excitación como la señal de respuesta en temperatura (ver Figura 6).

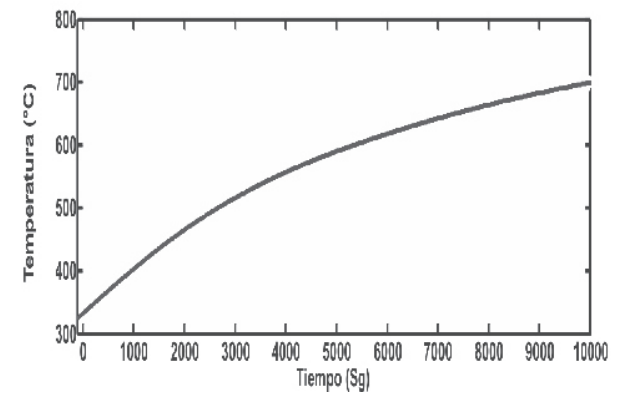

Figura 6. Curva de reacción del sistema ante un estímulo en la entrada. 
Obtenida la respuesta del sistema, y, por inspección, se deduce que esta es de un sistema de primer orden. Se utilizaron dos puntos sobre la curva de reacción, según lo propuesto por Smith (1972). Este seleccionó los tiempos para la identificación como los necesarios para alcanzar el 28,4 y el 63,2 \% (ver Figura 7), del cambio total en la respuesta a una entrada escalón (Alfaro, 2006).

$$
G(s)=\frac{0.9}{4050 s+1}(1)
$$

En la ecuación (1), se obtiene el modelo aproximado del sistema. Cabe resaltar que se desprecia el retardo presente en la planta, debido a que este es de un valor muy pequeño respecto al valor del $\underline{t}$. Para validar esta aproximación, se superpone el modelo obtenido de color magenta y la respuesta real de la planta de color azul, en la Figura 7.

$$
\begin{aligned}
& \dot{X}=A x+B u \\
& y=C x+D u \\
& \dot{x} 1=\frac{-1}{4050} x 1(t)+\frac{0.9}{4050} u(t) \\
& y(t)=[1] x_{1}(t) \\
& o b s=\left[\begin{array}{c}
C \\
C A \\
\cdot \\
\cdot \\
\cdot \\
C A^{2}
\end{array}\right]=1 \\
& \text { cont }=\left[\begin{array}{llll}
B & A B & \ldots \ldots & A^{2} B
\end{array}\right]=1
\end{aligned}
$$

Obtenidas las matrices de observabilidad y controlabilidad (ecuaciones 4 y 5), se puede afirmar que el sistema es completamente observable y completamente controlable (Balseiro, 2011; Fernández, 2011).

\section{DISEÑO DEL CONTROLADOR}

\subsection{Diseño del controlador PI}

Encontrado el modelo de la planta y verificada la estabilidad de la misma, se plantea el diseño del controlador de tipo PID, debido a que se utilizó el PLC Moller que se encuentra en el módulo didáctico EC4P, el cual en sus funciones internas cuenta con un bloque de control PID.

Dentro de las restricciones especificadas por parte del laboratorio de carbones, que deben tenerse en cuenta para diseñar el controlador de temperatura, está la que señala que la mufla no debería superar la temperatura seleccionada para evitar alteraciones en las muestras bajo estudio.

Otra restricción encontrada durante el desarrollo de algunas pruebas realizadas al sistema, fue el tiempo prolongado que le toma a la mufla disipar el calor debido a su construcción física de material refractario y aislante, lo que hace que tome alrededor de tres veces más de tiempo bajar la temperatura en comparación con el tiempo que le tomó subirla hasta ese mismo punto. Por tal razón, para el modelo deseado se eligió un tiempo de estabilización $t s$ igual al de la planta, a fin de no exigir un comportamiento exagerado en la respuesta del sistema, que solo conllevaría a excesivas señales de control además de contribuir a la saturación de la misma.

Haciendo:

$$
\begin{gathered}
\mathrm{ts}=\mathrm{t}=4050(6) \\
\rho=1.2(7) \\
\mathrm{ts}=\frac{4.6}{\rho^{*} \mathrm{wn}}(8) \\
\mathrm{Gd}(\mathrm{S})=\frac{\mathrm{wn}^{2}}{\left(\mathrm{~S}^{2}+2 * \rho^{*} \mathrm{wn}+\mathrm{wn}^{2}\right)}
\end{gathered}
$$

Remplazando las ecuaciones (6) y (7) en (8) y despejando.

Se obtiene que $\mathrm{wn}=0.00036(10)$

Remplazando de nuevo (10) en (9) se obtiene el modelo deseado para el comportamiento de la temperatura en la mufla (ver Figura 8)

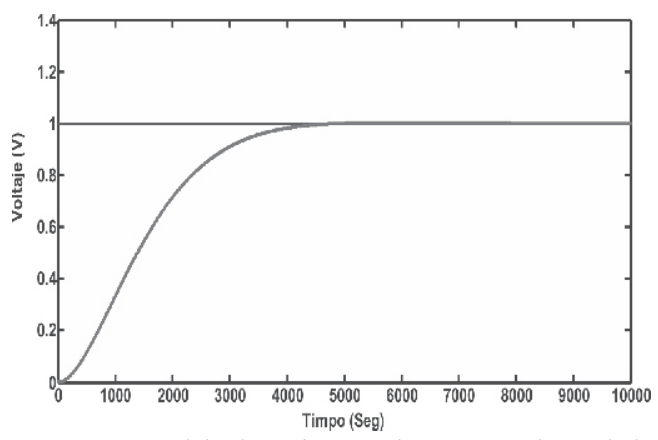

Figura 8. Modelo deseado para el comportamiento de la temperatura en la mufla.

Como se mencionó anteriormente, el controlador propuesto fue un PID. Ya que el elemento derivativo no es necesario para el sistema actual, cuya función de transferencia general para un controlador PI se muestra en (12).

$$
\frac{\mathrm{U}(\mathrm{S})}{\mathrm{E}(\mathrm{S})}=\mathrm{K}_{\mathrm{p}}+\frac{\mathrm{Ki}}{\mathrm{S}}(12)
$$

Igualando los polinomios del modelo deseado (ecuación 11), con la función de transferencia del controlador PI (ecuación 12), se obtiene la constante proporcional (ecuación 13) y la constante integral (ecuación 14).

$$
\mathrm{kp}=8.22(13)
$$$$
\mathrm{ki}=0.000615 \text { (14) }
$$

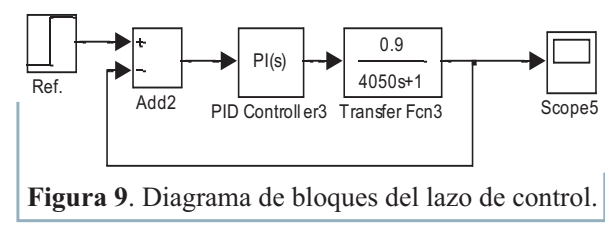

Obtenidas las constantes para el controlador PI, se procede a realizar la simulación del controlador con el sistema en lazo cerrado y observar el comportamiento de este (ver Figuras $10 \mathrm{y}$ 11). 


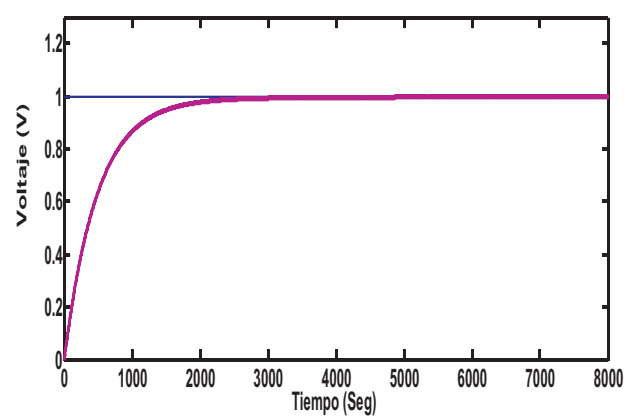

Figura 10. Respuesta del sistema aplicando el controlador

En la Figura 11 se observa en simulación la señal de control generada por el controlador al seguir la referencia, cumpliendo con la restricción propuesta al inicio del diseño de no generar señales de control demasiado grandes.

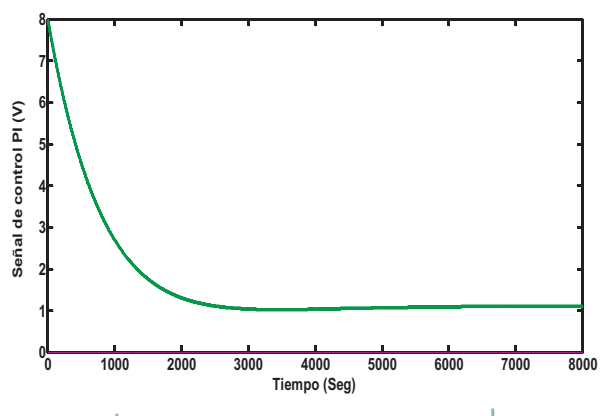

Figura 11. Señal de control

\subsection{Programación PLC}

Para realizar la programación del controlador PI en el PLC Moller, se utilizó el software de programación Easy Soft Codesys versión 2.3.9, explorando las configuraciones de la función interna PID del PLC. Al hacer la adquisición de la señal proveniente del transmisor de temperatura por la entrada analógica del PLC, es necesario escalizarla a unidades de ingeniería por medio de la función SCALE_R, disponible en el set de instrucciones del mismo. En el diagrama de flujo de la Figura 12 se muestra el proceso de programación efectuado para el PLC.

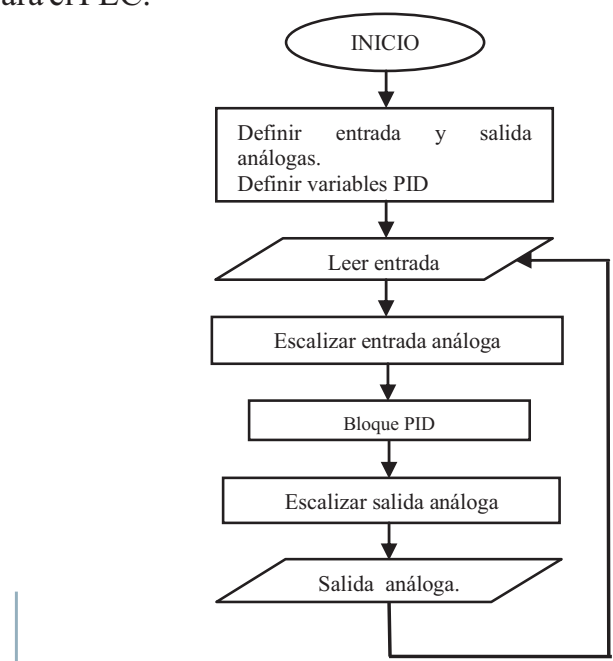

Figura 12. Diagrama de flujo de la programación realizada en el PLC.

\subsection{Visualización}

Utilizando la pantalla táctil HMI presente en el modulo EC4P, se hizo una sencilla visualización para la configuración y supervisión del proceso de temperatura, mediante cuatro pantallas enlazadas entre sí. La pantalla principal de supervisión se observa en la Figura 13, donde se detallan aspectos relacionados con la temperatura, porcentaje de uso de la variable de control, así como las opciones de configuración de los parámetros del controlador PID (ver Figura 14), configurar una rampa de temperatura programada previamente y observar gráficamente la temperatura de referencia y la temperatura actual del proceso. Cada visualización en su parte inferior presenta la opción de navegar entre las pantallas y tener un completo manejo del sistema de temperatura, así como un botón de encendido y apagado de todo el sistema de control.

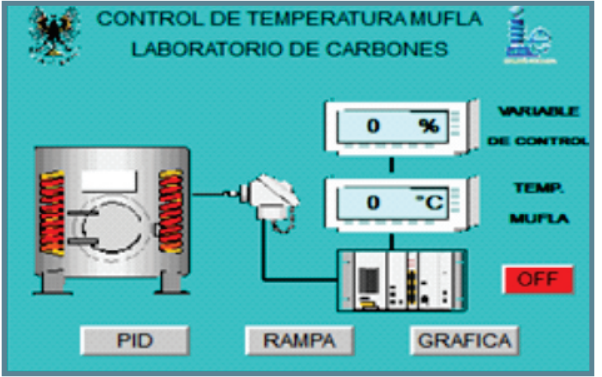

Figura 13. Pantalla principal del programa en la pantalla HMI para la supervisión del sistema de temperatura.

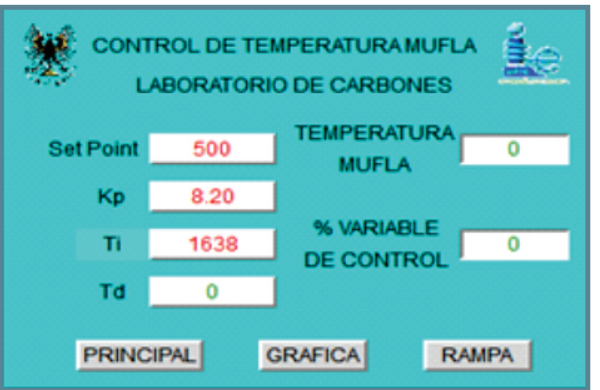

Figura 14. Pantalla de configuración de los parámetros del controlador PID

\subsection{Rampa de temperatura}

Acudiendo a sugerencias realizadas en el laboratorio de carbones, se programó una pequeña rampa de temperatura dentro de los rangos de operación del controlador. La rampa de temperatura parte desde los $400^{\circ} \mathrm{C}$ hasta los $450^{\circ} \mathrm{C}$; después de alcanzar ese valor, permanece en este durante 30 minutos. Pasados los 30 minutos, se da un incremento hasta los $500^{\circ} \mathrm{C}$ y permanece en este valor durante 60 minutos más. En la Figura 15 se muestra la pantalla programada en la HMI para la opción de rampa de temperatura.

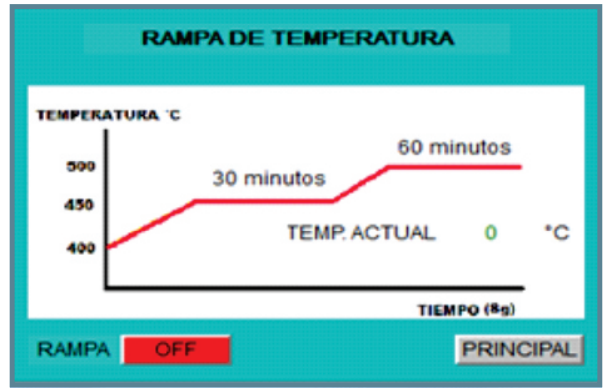

Figura 15. Pantalla programada para el manejo de la rampa de temperatura en la HMI. 


\section{RESULTADOS}

Efectuado el diseño del controlador y programado el PLC del módulo, se procede a realizar las pruebas del controlador directamente sobre la planta, fijando una temperatura deseada en el rango de $\operatorname{los} 320^{\circ}$ a los $700{ }^{\circ} \mathrm{C}$, donde se modeló el sistema.

Para poder observar de manera adecuada los resultados, se escalizó la información proveniente del sensor, debido a que la señal se tomó a la salida del transmisor de temperatura en voltaje y no se contó con la curva característica de conversión de voltaje a grados centígrados del transmisor. Para ello se utilizó la función que se observa en la ecuación (13), la cual se encontró de manera experimental al efectuar mediciones sobre el transmisor al variar la temperatura, donde $X$ es la señal en voltaje proveniente de la salida del transmisor, y $Y$ la salida en grados centígrados.

$$
\mathrm{y}=170.83 * X-305.66
$$

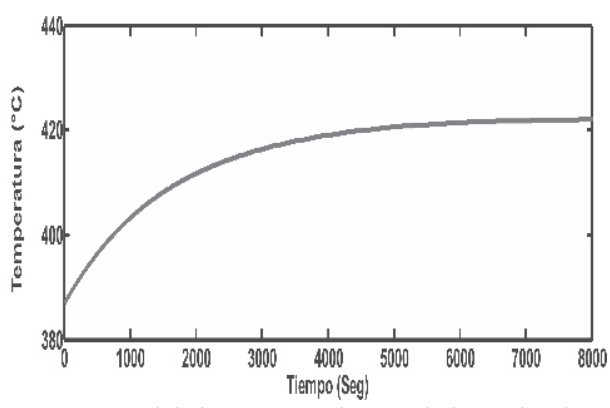

Figura 16. Respuesta del sistema con el controlador PI implementado.

Como se observa en la Figura 16, el sistema alcanza y mantiene la temperatura de referencia de $420^{\circ} \mathrm{C}$ en un tiempo aproximado de 4000 segundos, tal y como se especificó en el proceso de diseño

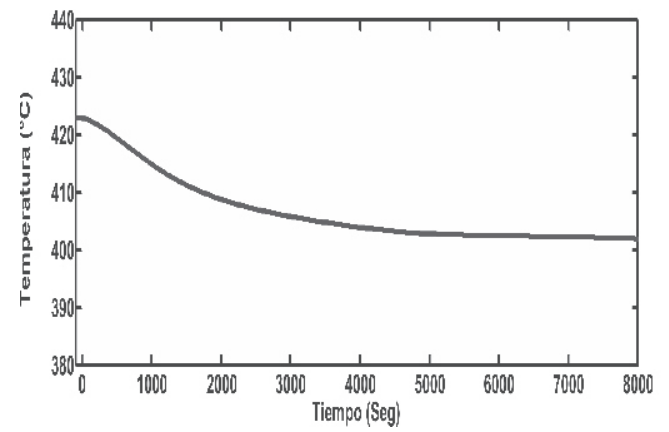

Figura 17. Respuesta del sistema controlado al variar la temperatura deseada.

Realizando otra variación a la temperatura de referencia, esta vez hacia una temperatura inferior, el sistema controlado nuevamente alcanza el valor de referencia y se estable en dicho valor, como se observa en la Figura 17. Otro aspecto importante en la verificacion de los resultados, es la señal de control real generada por el controlador (ver Figura 18), donde se observa que esta señal se encuentra dentro de los rangos del acondicionamiento efectuado para este $(0$ a $10 \mathrm{~V})$, sin llegar a saturarse, como se propuso en la fase de diseño y cumpliendo con la restriccion de no generar señales de control exageradas.

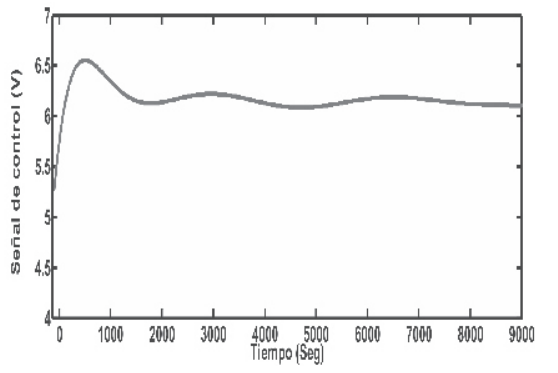

Figura 18. Señal de control generada por el controlador PI

Aplicando la función rampa de temperatura programada previamente en el módulo, en el rango de los 400 a $500{ }^{\circ} \mathrm{C}$, y adquiriendo esta señal mediante la tarjeta de adquisición de datos, se obtiene la rampa de la Figura 19.

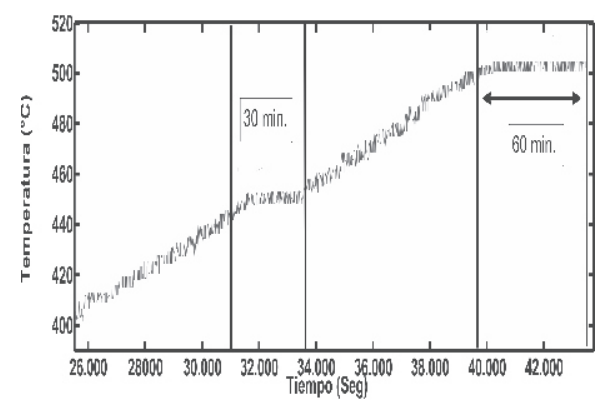

Figura 19. Curva obtenida de la rampa de temperatura programada.

\section{CONCLUSIONES}

El controlador PI diseñado e implementado sacrifica velocidad de respuesta del sistema para cumplir con la restricción propuesta por parte del laboratorio de carbones de no superar el valor de temperatura deseada y evitar el posible deterioro de las muestras bajo estudio.

El controlador diseñado e implementado reduce el tiempo general del proceso, ya que no supera el valor de la temperatura de referencia, sustentado en el hecho que debido a su construcción física, la mufla presenta una baja disipación térmica llegando a tomar hasta tres veces más tiempo bajar la temperatura, en comparación con el tiempo que le toma aumentarla desde una temperatura más baja.

El controlador diseñado e implementado para el sistema de temperatura como se propuso en la fase de diseño, no genera señales de control excesivamente grandes, lo que evita la saturación de este para llegar a la referencia deseada.

El uso de la pantalla HMI del módulo EC4P, permite una configuración sencilla del sistema de control y brinda la posibilidad de cambiar los parámetros de configuración del controlador y de las rampas de temperatura, sin necesidad de reprogramar de nuevo el PLC, además ofrece una supervisión completa de todo el sistema de control.

La rampa de temperatura programada ofrece otro nivel de aplicación al sistema de control, que permite versatilidad en los procesos térmicos, así como también la posibilidad de simplificar los estudios realizados en el laboratorio de carbones, 
ya que facilita la programación de rampas manipulando parámetros, como la temperatura deseada, tiempo de ascenso y tiempo de permanencia de esta, según los requerimientos del laboratorio.

\section{REFERENCIAS}

Alfaro, V. (2006) Identificación de modelos de orden reducido a partir de la curva de reacción del proceso.

Costa Rica:

Departamento de Automática, Escuela de Ingeniería Eléctrica Universidad de Costa Rica. Recuperado $\mathrm{d}$ http://eie.ucr.ac.cr/uploads/file/documentos

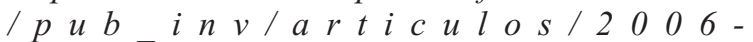
CyT.VAlfaro.cyt.v24n2_2006.pdf

Balseiro, I. (2011). Controlabilidady observabilidad

Control digital.

Argentina. Recuperado de

http://www.ib.cnea.gov.ar/ control2/Links/

Apuntes/CDVE-Cap5.pdf

Bañuelos, M., Castillo, J., Rayo, G., Quintana, T., Damián,

R. \& Pérez, J. Controlador

Pid de temperatura de tipo didáctico.

M é x i c o : U N A M . R e c u p e r a d o d e http://proton.ucting.udg. $\mathrm{mx} /$ somi/memorias ldidactica/Did-2.pdf

BPA. (2011). Manual módulo didáctico PLC EC4P. s.1.: s.n.

Chapman, J. (1993). Máquinas eléctricas. (2ª ed.). Colombia: McGraw Hill.

Dolores, R., Barber, M. \& Salichs, M. (2012).

Lugar delas raíces. Madrid: Universidad Carlos IIIde $\mathrm{M}$ a d r i d. R e c u p e r a d o d e http://ocw.uc3m.es/ingenieria-de-sistemas-yautomatica/senales-y-sistemas/temas/tema-13-

Espinoza, J. (2010). Apuntes sistemas de control - 543244. Control automático UDEC. (10ed.).Universidad de Concepción Facultad de Ingeniería, Depto. de concepción Facultad de Ingeniería, Depto. de Ingeniería Eléctrica. Chile. Recuperado de http://www2.udec.cl/ joseespi/SDC/543244_SDC_ Apuntes.pdf

Fernández, L. (2010). Apuntes de clase. Sogamoso:

Escuela de Ingeniería Electrónica.

Fernández, L. (2004). Libro apuntes de clase.

Sogamoso: Escuela de Ingeniería Electrónica.
Herrera, J., Vásquez, L. \& Estalin, A. (2011). Diseño, programación e instalación de un sistemade control de supervisión y adquisición de datos de un horno Lindberg para el laboratorio de tratamientos térmicos. Quito: Escuela

Politécnica Nacional. Recuperado de http://bibdigital.epn.edu.ec/bitstream/15000/3993/1/CD3765.pdf

Intech. (2011).Xu transmitter series. Recuperado de http://www.intech.co.nz/products/transmitters/xu2/XU sales.pdf

Luengo, R. (2003). Cerámica: tipos de hornos.

Recuperado de

http://www.xtec.cat/ aromero8/hornos.htm

Martínez, P. \& Azuaga, M.(1997). Calibración de una Termocupla de Chromel-alumel. Recuperado de http://www.investigacion.frc.utn.edu.ar/sensores/ temperatura/termocuplas.pdf

Ogata, K. (1998). Ingeniería de control moderna. México: Pearson Education.

Salamanca, J. (2011). Apuntes de clase. Sogamoso: Escuela de Ingeniería Electrónica.

Semikron. (2004). Thyristor/Diode Modules SKKT 15 SKKH. Recuperado de http://www.semikron.com/products/data/cur/assets/ SKKT_15_07233361.pdf

Smith, C. (1972). Digital Computer Process Control. EUA: International Textbook, Co.

Tarazona, J. (2008). Diseño y construcción de un controlador

de temperatura tipo rampa para el Laboratorio de Catálisis-UNET. Venezuela: Universidad Nacional Fxperimmentatedel.uTáchiua: Requbparexarder/db/bcunet/ edocs/TEUNET/2008/pregrado/Electronica/Tarazona D_JacksonL/Preliminares.pdf 